\title{
TISSUE ENGINEERING IN MAXILLOFACIAL BONE RECONSTRUCTION
}

\author{
David B. Kamadjaja ${ }^{1}$, Purwati ${ }^{1}$, Ferdiansyah $^{1}$, Fedik Abdul Rantam $^{1}$
}

${ }^{1}$ Stem Cell Research and Development Center, Universitas Airlangga Surabaya, East Java, Indonesia

\begin{abstract}
Maxillofacial bone defects due to tumor resection, trauma or infections should be reconstructed to maintain the bone continuity in order to preserve its masticatory, speech and esthetic functions. Autogenous bone graft have been the gold standard for mandibular defects reconstruction, however, it is associated with limitation in volume and availability as well as the donor site morbidities. Tissue engineering approach has been proved to be a good alternative to overcome the limitation of autogenous bone graft. Tissue engineering studies have been conducted combining various sources of mesenchymal stem cell, scaffolds, and or signaling molecules. The paper aims to provide information on the development of bone tissue engineering researches to reconstruct bone defects through results of numerous studies obtained in the English literature. As the conclusion, bone tissue engineering is a potential approach to reconstruct maxillofacial bone defects.
\end{abstract}

Keywords: scaffold,osteoconduction, mesenchymal stem cell, bone regeneration, bone integration.

\section{Introduction}

Surgical reconstruction of critical size mandibular defect has been a great challenge in oral and maxilla facial surgery. Gold standard for mandibular reconstruction is autogenous bone graft. However, autogenous bone grafthas limitation in shape, size, and its availability; furthermore, it has been attributed to donor site morbidity (Arrington et al., 1996; Heary et al., 2002). While the transfer of autologous tissue such as bone grafts or tissue free flaps are welldescribed, they are not without complications.To overcome these limitation, one can expect more on bone tissue engineering. Tissue engineering or tissue regeneration is a multidisciplinary approach to replace tissue loss as a result of traumatic defects, tumor resection or infection (Srisuwan et al., 2000). The prospect of using principles of tissue engineering to reconstruct defects in oral and maxillofacial defects continues to gain the attention of the reconstructive surgeon.(Susarla et al., 2011)Numerous studies in bone tissue engineering have attempted to search for effective combination of stem cells, scaffolds, and signaling molecules for reconstruction of bone defects in various animal models. A tissue engineering approach provides numerous prospective benefits, including a decline in donor site morbidity, a decrease in procedural sensitivity of the repair, and the capacity to intimately mimic the in vivo tissue environment 
into recapitulate normal craniofacial development (Ward et al., 2010).The paper aims to provide information on the development of tissue engineering to reconstruct maxillofacial bone defects.

\section{Principles of tissue engineering}

Principles of tissue engineering are basically a triad of stem cells, signaling molecules, and scaffolds or extracellular matrix (Figure 1). (Rai et al., 2015). A stem cell is defined as an unspecialized cell that can renew and maintain itself for a longer period of time with the potential to commit to a cell or tissue lineage with specialized functions. The use of stem cells, either embryonic or adult-derived (ADSCs), is a reality of regenerative medicine and dentistry. ADSCs are multipotent cells not derived from embryonic or primordial germ cell lineage, and they have the potential to differentiate into bone, muscle, cartilage, nerve, and vasculature under appropriate conditions (Conrad et al., 2005). These stem cells can differentiate into various cells like chondrocytes, osteoblasts, myoblasts, hematopoietic cells, and neural cells (Figure 2).

Signaling molecules: Various growth factors and cytokines are mixed to the ECM, like bone morphogenetic proteins (BMP), fibroblast growth factor-2 (FGF-2), interleukin-6, insulinlike growth factor (IGF), platelet-derived growth factor (PDGF), transforming growth factor- $\beta 1$, etc. This co-localization works as a storage pool of growth factors and may diminish growth factor degradation, protecting them from the local micro environment while facilitating the presentation of the growth factors to cell surface receptors (Schnaper et al., 1993; Roberts et al., 1988).

Scaffold: A scaffold is a permanently or temporarily placed three-dimensional porous and permeable natural or synthetic biomaterial that is biocompatible. It can be natural (Figure 2) or synthetic. It acts as a matrix and allows the attachment, migration, and differentiation of progenitor cells. Properties of scaffolds (such as biodegradability, porosity, stiffness, and strength) influence cell adhesion, migration, and proliferation (such as osteoconduction). The greatest challenges faced in tissue-engineered devices, regardless of tissue type, is promoting healing in three dimensions. Scaffolds have been made-up with a variety of innate and synthetic biomaterials, such as ceramics, metals, proteins, and polymers. An appropriate scaffold for tissue engineering will be one that is created with biology in mind. The functionof the scaffolds is providing structural support to cells, reservoir for growth factors and provide flexible, physical environment for remodeling (Patil et al., 2013). 
Only 2 pages have been converted.

Please go to https://docs.zone and Sign Up to convert all pages. 